\title{
Functional Repair of the Corticospinal Tract by Delayed Transplantation of Olfactory Ensheathing Cells in Adult Rats
}

\author{
Naghmeh Keyvan-Fouladi, Geoffrey Raisman, and Ying Li \\ Division of Neurobiology, Norman and Sadie Lee Research Centre, Medical Research Council, National Institute for Medical Research, London, NW7 1AA, \\ United Kingdom
}

\begin{abstract}
Adult rats were trained to use their forepaws to retrieve a piece of food. Destruction of the dorsal corticospinal tract on one side at the level of the first cervical segment abolished the use of the ipsilateral forepaw for retrieval for at least 6 months after operation. Where a variable amount of the corticospinal tract was spared, there was a proportionate persistence of retrieval. In lesioned rats that had shown no retrieval for 8 weeks after operation, a suspension of olfactory ensheathing cells was injected into the lesion site. Starting between 1 and 3 weeks after transplantation, all rats with transplants bridging the lesion site resumed retrieval by the ipsilateral forepaw. Biotin dextran anterograde tracing shows regenerating corticospinal axons crossing the bridge, traveling caudally for $\sim 10 \mathrm{~mm}$ in the distal part of the corticospinal tract and forming terminal arborizations in the spinal gray matter. Functional recovery can occur when only $\sim 1 \%$ of the corticospinal tract axons are present.
\end{abstract}

Key words: spinal cord; axon; lesion; CNS; function; transplantation; OECs; delayed; regeneration; adult; repair

\section{Introduction}

We have reported previously that unilateral lesions of the dorsal corticospinal tract (CST) abolish directed forepaw retrieval (DFR) by the ipsilateral forepaw (Li et al., 1997). Olfactory ensheathing cells (OECs) transplanted into the lesion site at the time of injury mediate a recovery of DFR. The potential value of such an approach to human spinal cord injuries will have to take into account the inevitable delay that will occur in clinical practice between the time of injury and the time when the patient is sufficiently stable, to be certain that no further spontaneous recovery can be expected without transplantation.

In most previous studies of repair, transplantation was performed at the same time the lesions were made (Imaizumi et al., 1998; Ramón-Cueto et al., 1998, 2000). More recently, functional improvement has been reported after delayed transplantation of fetal spinal cord, cerebral tissue, and adult peripheral nerve tissue (Coumans et al., 2001; Zurita et al., 2001) into chronic rat spinal cord lesions. Lu et al. (2002) and Plant et al. (2003) have reported an improvement of locomotor function after delayed transplantation of OECs into complete or incomplete thoracic spinal lesions. In the present study, we investigate whether OECs are still able to restore DFR when transplantation is delayed for 2 months after complete unilateral destruction of the CST.

\section{Materials and Methods}

Pretraining. On 3 successive days before operation, adult (body weight, 200-240 gm) female rats of a locally inbred Albino Swiss strain were

Received June 27, 2003; revised Aug. 20, 2003; accepted Aug. 28, 2003.

This work was supported by the British Neurological Research Trust and the International Spinal Research Trust. Dr. Pauline Field provided important collaboration.

Correspondence should be addressed to Dr. Geoffrey Raisman, Division of Neurobiology, National Institute for Medical Research, The Ridgeway, Mill Hill, London, NW7 1AA, UK. E-mail: graisma@nimr.mrc.ac.uk.

Copyright $\odot 2003$ Society for Neuroscience $\quad$ 0270-6474/03/239428-07\$15.00/0 introduced to the testing cage, a piece of food was presented through a slit in the front of the cage, and the number of times the right and left paws were used was scored up to an overall total of 50 retrievals. The majority of rats use both paws more or less equally. Less than $10 \%$ of rats showed an asymmetry of use $>15 / 35$, and these were not used further.

Lesion. Because we wanted to study the functional effect of unilateral lesions of the CST and reduce involvement of other tracts in the dorsal columns, as well as avoiding damage to the lateral columns, we used a stereotaxic approach in which we have attempted to confine the lesion size and position to the CST. Anesthetized rats $(n=54)$ were mounted in a stereotaxic frame. With the neck flexed, the dorsal surface of the spinal cord was exposed at the level of the first to second cervical segments through a midline incision. A stainless steel electrode of $250 \mu \mathrm{m}$ diameter, insulated to within $1 \mathrm{~mm}$ of the tip, was inserted to a depth of $1 \mathrm{~mm}$ at a point $500 \mu \mathrm{m}$ lateral to the midline. The identification of the midline depends mainly on the presence of the median sagittal vein. However, this is not absolutely consistent, leading to some variation in electrode placement. Approximately equal numbers of rats were operated on the right and left sides. The CST was destroyed by a heat lesion produced by a radiofrequency lesion device (RFG-3C RF; Radionics Inc., Burlington, MA) operating at $10 \mathrm{~V}$ for $1 \mathrm{~min}$. This produces circumscribed ovoid lesions of $0.5-1.0 \mathrm{~mm}$ in diameter. Because the size of the lesions (0.5-1.0 $\mathrm{mm})$ was not much greater than that of the target CST $(0.5 \mathrm{~mm})$, slight errors in electrode placement led to sparing of varying amounts of the target.

Postoperative testing. Three days after operation, the rats were introduced to the testing cage and the use of the right and left paws was scored for 50 retrievals three times per week for 8 successive weeks. In 21 rats that showed either retention of ipsilateral DFR or a loss of DFR, followed by a progressive return, subsequent histology showed that in all cases the lesions of the CST were incomplete. This series of incomplete lesions was used to study the correlation between lesion size and DFR. Of 33 rats that did not use the ipsilateral forepaw for retrieval for the 8 weeks of postlesion testing, 7 were killed, and the completeness of the lesions was verified by calcium calmodulin kinase II (CaM-II) immunostaining (see below). Of the remaining 26 rats, 12 received transplants of OECs (see 

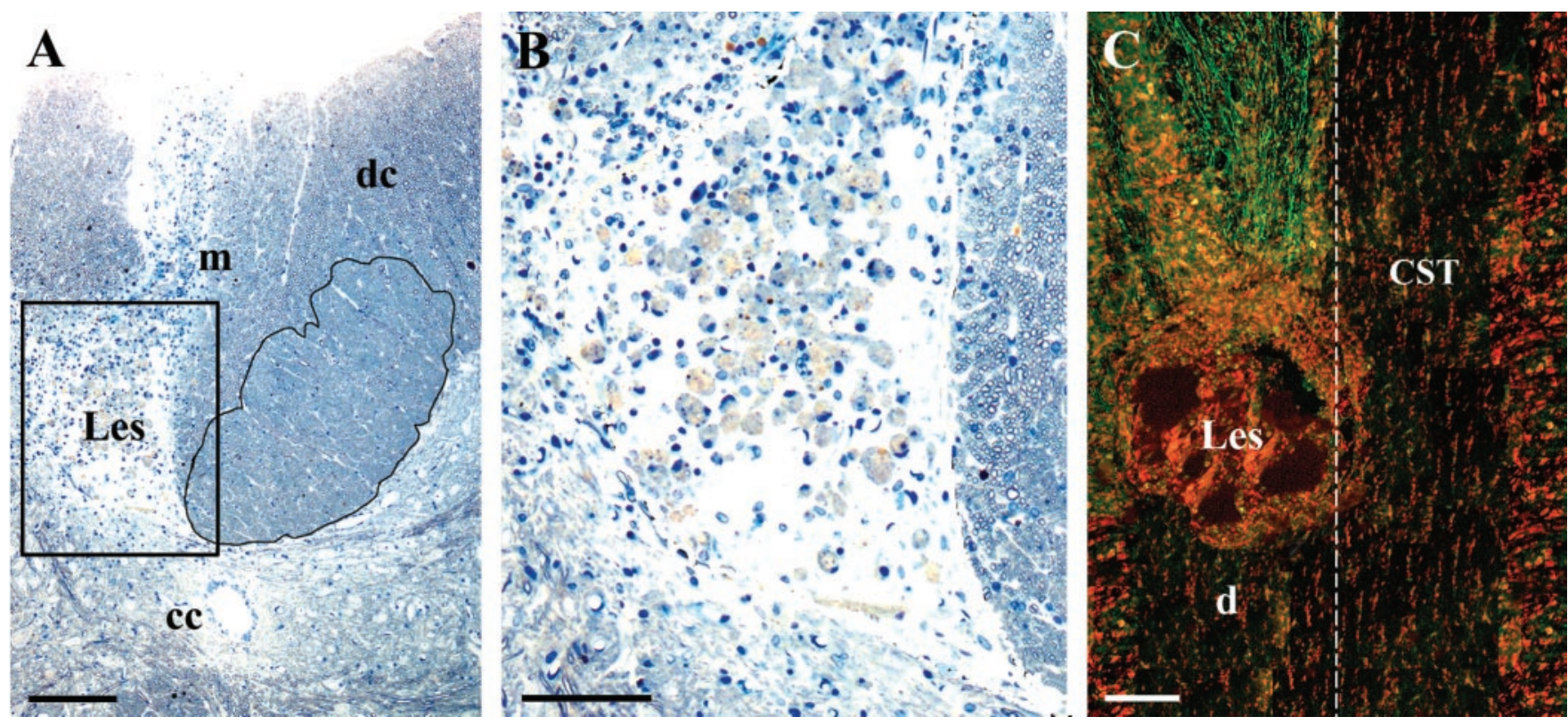

Figure 1. A, A two micrometer semithin cross-section showing the intact CST (right, outlined) and complete destruction of the left CST (Les, filled with macrophages). cc, Central canal; dc, dorsal columns (ascending fibers); $m$, midline. The boxed area is enlarged in $B$ and stained with methylene blue and Azur Il. Survival time is 2 months. C, Confocal image of horizontal $60 \mu \mathrm{m}$ section showing BD anterograde-labeled CST axons (green) rostral to the lesion (Les) on the left and complete absence of axons in the left CST caudal to the lesion (d). The contralateral CST is unlabeled because the BD was only applied to the ipsilateral cortex. Dashed line, Midline. The counterstain is propidium iodide (red). Survival time is 6 months. Scale bars: $A, 200 \mu \mathrm{m} ; B, 50 \mu \mathrm{m} ; C, 100 \mu \mathrm{m}$.

below) and 2 received injections of OEC-conditioned medium (as a control). These rats were tested three times per week for an additional 8 weeks. Of the remaining 12 lesioned rats (without transplants), six were tested once a week for an additional 16 weeks (to a total of 24 weeks).

Cell culture. Tissue was dissected from the outer nerve and glomerular layers of the rat olfactory bulb, trypsinized $(0.1 \%$ trypsin for $15 \mathrm{~min}$ at $37^{\circ} \mathrm{C}$ ), plated onto $35 \mathrm{~mm}$ dishes coated with poly-L-lysine, and cultured for 14-17 d in DMEM-F-12 medium including 10\% FCS (3133-028; Invitrogen, Paisley, UK). Each dish yielded $\sim 1$ million cells, of which $\sim 50 \%$ were 75 -positive, Schwann-like OECs and 50\% were fibronectin (FN)-positive olfactory nerve fibroblasts (Li et al., 1998).

Transplantation. Fourteen rats that had shown complete absence of DFR for 8 weeks after lesion were reanesthetized, and the spinal cord was reexposed. Using the same coordinates as for the lesioning, a solenoidcontrolled air pressure device injected $5 \mu \mathrm{l}$ of a suspension of $25 \times$ $10^{6} / \mathrm{ml} \mathrm{OECs}(n=12)$ or OEC-conditioned medium $(n=2)$ via a glass micropipette (100-150 $\mu \mathrm{m}$ tip) into the lesion site (Li et al., 1997).

Axon tracing. Four rats with transplants and six rats with lesion alone were used for tracing CST axons. A total of $10 \%$ biotin dextran (BD) $(0.5$ $\mu \mathrm{l}$; molecular weight, 10,000; Molecular Probes, Eugene, OR) (Brandt and Apkarian, 1992) in saline was injected stereotaxically under anesthesia through a $100 \mu \mathrm{m}$ tip glass micropipette at each of the four points along the mediolateral extent of the contralateral sensorimotor cortex $(\mathrm{Li}$ et al., 1998) 2 weeks before perfusing with $4 \%$ paraformaldehyde.

Histology. For immunostaining on semithin sections, rats were perfused under deep terminal anesthesia with a mixture of $1 \%$ paraformaldehyde and $1 \%$ glutaraldehyde in $0.1 \mathrm{M}$ PBS and embedded in resin. Coronal sections (1-2 $\mu \mathrm{m}$ ) were cut through the lesion/transplant site, treated with ethanolic sodium hydroxide for $9 \mathrm{~min}$ for resin etching, and immunostained on a slide with 1:1000 anti-FN (Invitrogen) or 1:3000 P0 antiserum (a gift from J. J. Archelos, Max-Planck Institute, Munich, Germany). The reaction product was visualized with diaminobenzidine, and the sections were counterstained with methylene blue and Azur II.

For BD labeling, rats were perfused with 4\% paraformaldehyde and 60 $\mu \mathrm{m}$ horizontal cryostat sections were cut through the lesion/transplant area and incubated in $\mathrm{ABC}$ (Vector Laboratories, Peterborough, UK) overnight. BD was visualized by 1:200 Strep Avidin-green (Alexa Fluor 438; Molecular Probes) in PBS for $2 \mathrm{hr}$ at room temperature, washed in PBS, and counterstained with propidium iodide for confocal microscopy.
To identify the transplanted cells and monitor cell migration, rats were perfused with PBS and $10 \mu \mathrm{m}$ horizontal cryostat sections were immunostained for FN (as above) and counterstained with $0.5 \%$ aqueous neutral red. For estimating the completeness of the lesions, $10 \mu \mathrm{m}$ coronal cryostat sections were immunostained with 1:500 CaM-II (Zymed, San Francisco, CA) (Terashima et al., 1994) and counterstained with $0.5 \%$ aqueous neutral red. Photomicrographs at $10 \times$ were taken of three adjacent sections at $1 \mathrm{~mm}$ caudal to the lesion and printed out. The extent of the lesions was expressed as a percentage of the area of the unoperated side (an average of the three sections), measured by placing a $5 \mathrm{~mm}$ squared grid over the CST.

\section{Results}

\section{Lesions without transplants}

The CST can be identified in methylene blue/Azur II-stained semithin sections (Fig. 1A). The corticospinal axons were characterized by anterograde BD labeling in serial horizontal sections (Fig. 1C) and CaM-II immunohistochemistry in cross-sections (Fig. $2 \mathrm{~A}$ ). At the top cervical level, the CST is $\sim 0.5 \mathrm{~mm}$ in diameter. The electrode produced lesions of $\sim 0.5-1.0 \mathrm{~mm}$ in diameter on one side. In semithin sections, the lesions were fluid-filled cavities containing macrophages and tissue debris but neither axons nor glia (Fig. $1 A, B$ ). The unavoidable minor variations in electrode positioning resulted in varying degrees of destruction of the CST (see Materials and Methods). The extent of the lesions was assessed by using semithin sections, by anterograde BD labeling (Fig. 1C) in a complete series of contiguous horizontal sections through the entire width of the tract, or by CaM-II staining in the tract $1 \mathrm{~mm}$ caudal to the lesion (Fig. $2 F$ ). In the case of incomplete lesions, the degree of destruction of the CST was expressed as the area of remaining CaM-II staining (Fig. 2).

\section{Functional effect of lesions}

Rats with no functional recovery

There were 19 rats that never used the ipsilateral forepaw for retrieval at any time during the postoperative period of 8 weeks $(n=7$; Fig. $3 A, \boldsymbol{\Delta})$ or 24 weeks $(n=6)$. In the six cases with 

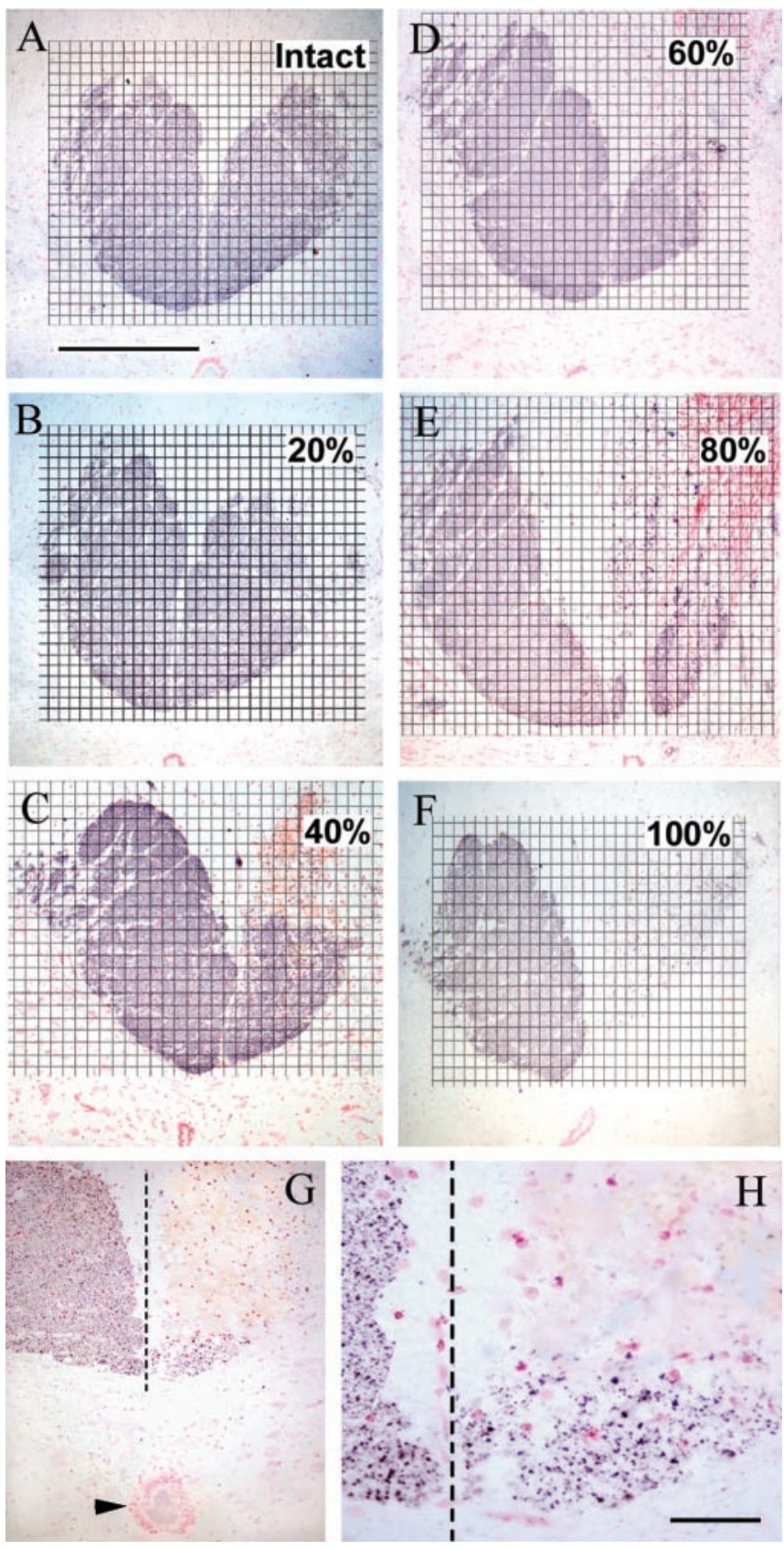

Figure 2. The extent of axon loss from the CST caudal to the lesions is assessed by placing a $5 \mathrm{~mm}$ squared grid over photomicrographs of CaM-Il $\alpha$-immunostained, $10-\mu \mathrm{m}$-thick crosssections through the distal CST $1 \mathrm{~mm}$ caudal to the lesion (to aid comparison, all lesions are shown on the right). The extent of the loss of CaM-II staining varies from none ( $A$, Intact), in five increasing steps of $20-100 \%$ ( $B-F)$ is shown. G, CST with $\sim 1 \%$ of the CaM-II-positive axons spared (area enlarged in $H$ has $\sim 400$ axons). Arrowhead, Central canal; dashed line, midline. The counterstain is neutral red. Survival time is 8 weeks. Scale bars: $A-G, 500 \mu \mathrm{m} ; H, 200 \mu \mathrm{m}$.

semithin histology, there was a cyst that occupied the entire position of the CST, leaving no detectable remnants of the tract on the lesioned side. In the seven cases with CaM-II staining, there was no visible staining in the distal tract. In the six cases with $\mathrm{BD}$ tracing, no fibers were observed in the lesion area or in the distal tract. In response to food presentation, it was clear that these rats attempted to use the ipsilateral forepaw, because repeated shortrange distal grasping movements (wrist supination and digit flexion) were performed in response to food presentation, but they were ineffective at retrieval because the animals did not use the

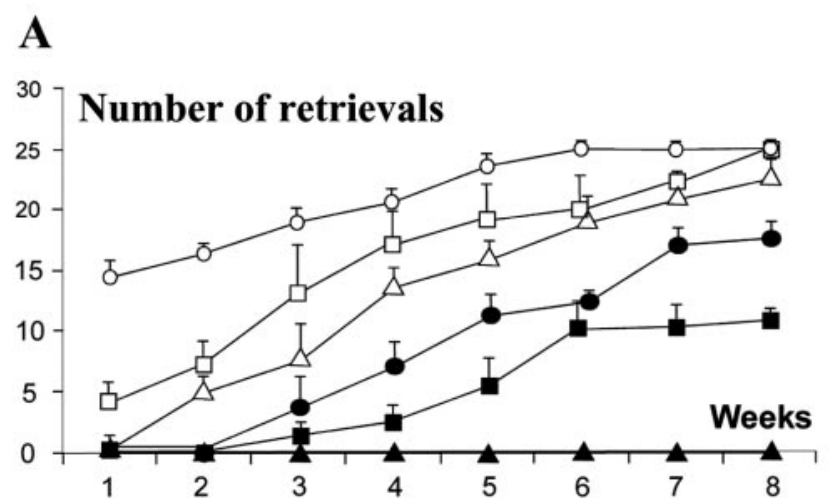

\section{B}

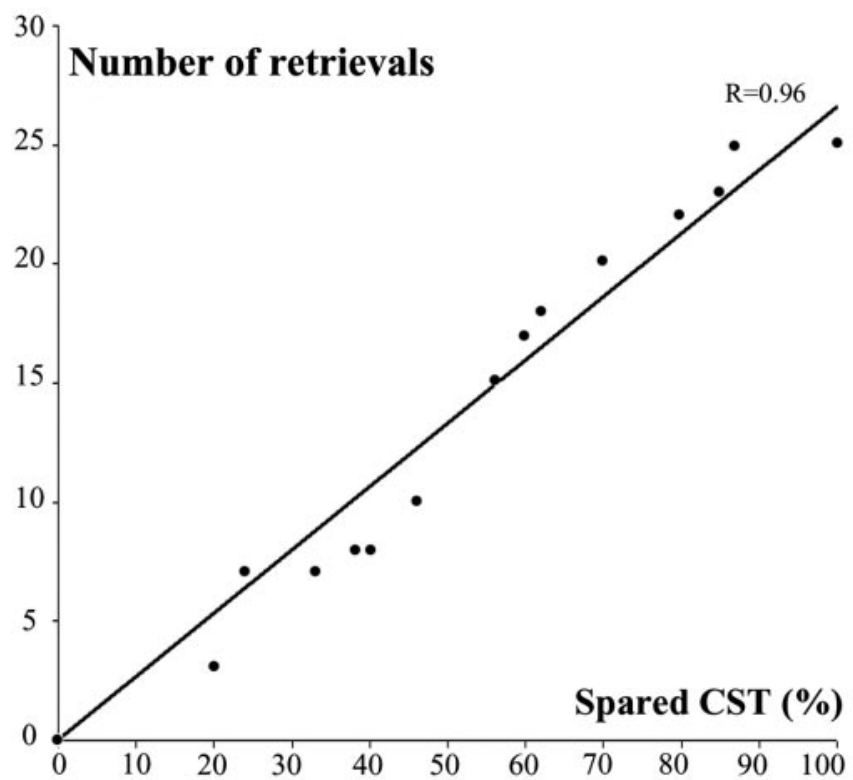

Figure 3. A, Changes with time in weeks ( $x$-axis) in the number of retrievals ( $y$-axis) performed by the forepaw ipsilateral to the lesion in each testing period (of a total of 50 retrievals by both forepaws). The rate of recovery of DFR over the 8 postoperative weeks is proportional to the amount of the CST spared by the lesion. The percentage destroyed is expressed in bins of

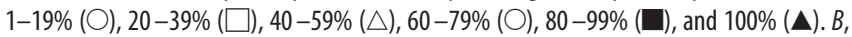
Significant correlation $(r=0.96)$ between the total number of retrievals by the ipsilateral forepaw ( $y$-axis) and the proportion of the CST spared ( $x$-axis).

proximal arm and shoulder muscles to achieve the directed movement of extending their arms through the slit, and so the grasp did not get within range of the food. Even when the contralateral forepaw was restrained, the rats were unable to perform retrieval with the ipsilateral forepaw and instead attempted to retrieve the food with the tongue or snout. Apart from the defect in retrieval, we found no impairment of the ipsilateral forepaw in other functions, such as walking along a cage edge, climbing, grooming, and picking up and handling food, all of which showed no asymmetry.

\section{Rats with functional recovery}

There were 21 rats that retained a degree of DFR by the ipsilateral forepaw. Subsequent histology showed that each had a degree of sparing of the CST fibers, as assessed by the presence of CaM-II immunostaining in the distal CST $1 \mathrm{~mm}$ caudal to the lesion. In the three groups of rats with the smaller lesions (destroying up to $60 \%$ of the tract), the ipsilateral forepaw was used from the first 


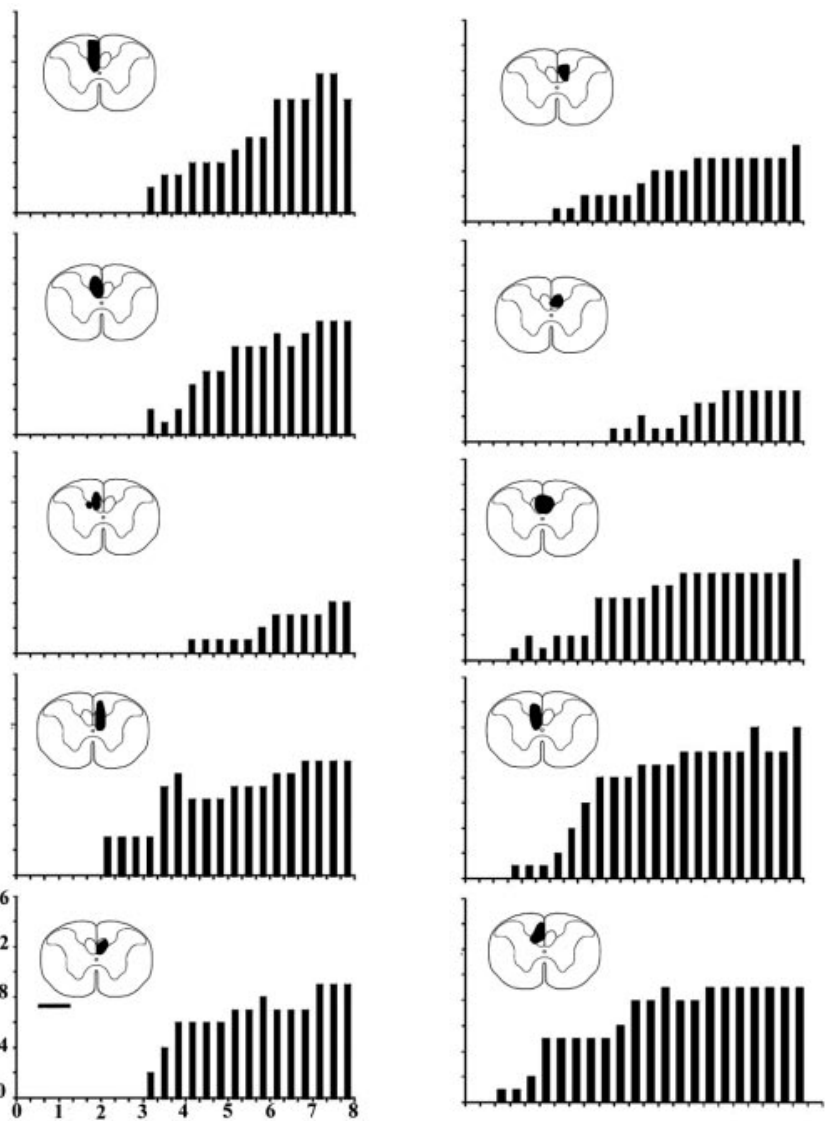

Figure 4. The onset and progressive increase in the numbers of paw retrievals ( $y$-axis) achieved in each of the thrice weekly tests over the 8 weeks ( $x$-axis) after transplantation in the series of 10 rats that had shown no DFR in the 8 week period after lesioning and preceding transplantation of OECs. The approximate size and position of the maximal extent of each of the transplants is indicated in solid black on the thumbnail outlines of the spinal cord. Scale bar, $1 \mathrm{~mm}$.

time tested, but at a reduced level, which increased progressively and reached close to normal levels by 8 weeks. The two groups of rats with the larger lesions (destroying $\geq 60 \%$ of the tract) did not start retrieval until the second postoperative week and were still severely impaired at 8 weeks, although the rising curve suggested that greater recovery might have been acquired at longer survivals. Lesions sparing only $\sim 1 \%$ of the CST axons (Fig. $2 G, H$, a case with $\sim 400$ axons) were still compatible with spontaneous return of DFR, although after a delay of 3 weeks. The data in Figure $3 \mathrm{~A}$ are displayed in bins of $1-19 \%(n=4$ rats $), 20-39 \%(n=4)$, $40-59 \%(n=4), 60-79 \%(n=5), 80-99 \%(n=4)$, and $100 \%$ $(n=7)$. Taken as a whole, the total number of retrievals by the ipsilateral forepaw is strongly correlated $(r=0.95)$ with the percentage of surviving axons (Fig. 3B).

\section{Transplants}

\section{Function}

Transplantations were performed in an additional group of 14 rats in which there had been no recovery of DFR for 8 weeks after lesion. Of these, 12 received transplants of OECs and 2 received control injections of OEC-conditioned medium. All 14 rats were then tested for DFR three times per week for 8 weeks after the transplantation. Of these, 10 showed a return of DFR, which began at 1-3 weeks after transplantation, and continued with some additional increase up to 8 weeks after transplantation (Fig. 4). Subsequent histology (see below) showed in all 10 of these rats
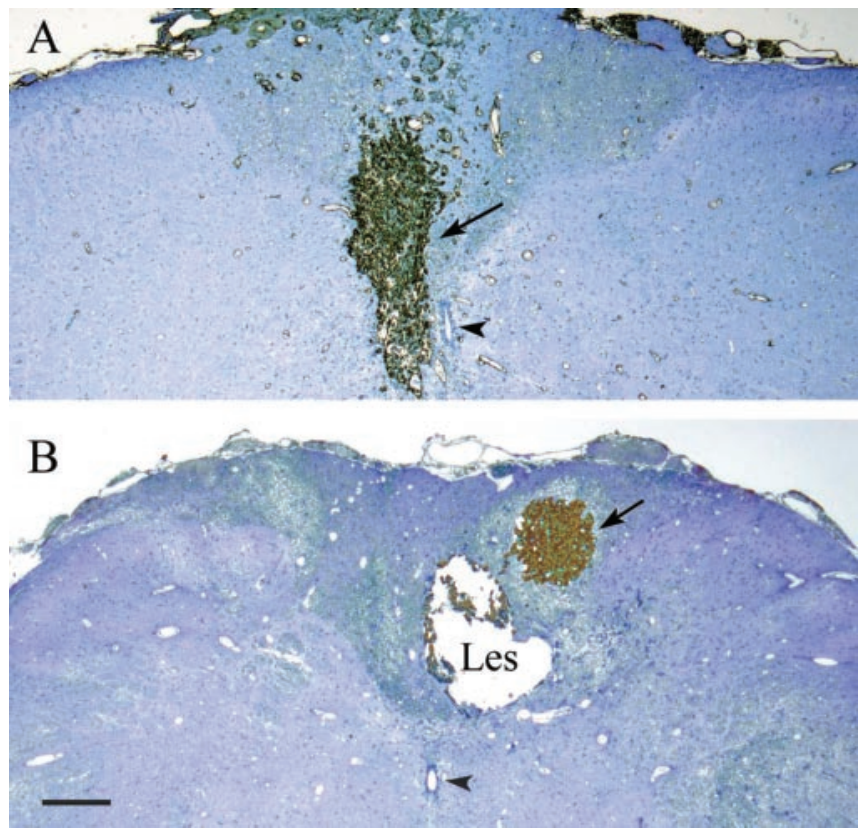

Figure 5. Cross-sections showing transplants (arrows) intensely stained with anti-FN antibody. In $A$, the transplant completely fills the original site of the lesion of the CST. In $B$, the injection was misplaced, and the transplant lies in the dorsal columns, without making contact with the cystic lesion (Les) of the CST. Two micrometer semithin sections are counterstained with methylene blue and Azur II. Arrowheads, Central canal. Survival times are 16 weeks after lesion and 8 weeks after transplant. Scale bar, $500 \mu \mathrm{m}$.

that the CST had been completely destroyed and the lesion site completely filled with transplanted OECs (Fig. 5A). That the recovery of function after this long lesion plus transplant survival period ( 16 weeks) was not simply attributable to the longer overall survival period (of $8+16=24$ weeks) or the prolonged testing is demonstrated by the failure of return of DFR in six untransplanted rats at 24 weeks after complete lesions (see above).

Two of the transplanted rats showed no return of DFR over the 8 weeks after transplantation. In these rats, subsequent histology showed that the transplants had been misplaced dorsally, in the ascending dorsal columns, and did not occupy the lesioned CST (Fig. $5 B$ ). The two rats with control injections of OECconditioned medium also showed no return of DFR over the 8 weeks after injection, and the histology in all four of these cases confirmed that the lesions had completely destroyed the CST.

\section{Histology}

FN staining of the olfactory nerve fibroblasts enabled the transplants to be identified clearly as solid, highly vascularized tissue masses, $\sim 0.5 \mathrm{~mm}$ in transverse diameter and elongated to $\sim 1.0$ $\mathrm{mm}$ in the dorsoventral direction (Fig. 5A). In the 10 rats in which the transplants were located in the CST, the transplanted cells filled the former lesion cavity and extended caudally for up to $7 \mathrm{~mm}$ in the distal part of the CST (Fig. 6A). p75 immunostaining, although useful for identifying the Schwann-like OECs in culture and at short-term survivals after transplantation, was not helpful in the present material because it is downregulated after $\sim 3$ weeks, although the presence of the Schwann-like OECs was later indicated by P0 immunostaining for peripheral myelin (Fig. 7; see below).

$\mathrm{BD}$ anterograde tracing data in four rats with transplanted OECs and post transplantation return of DFR showed BD-labeled CST axons entering and traversing the transplants. After crossing the le- 

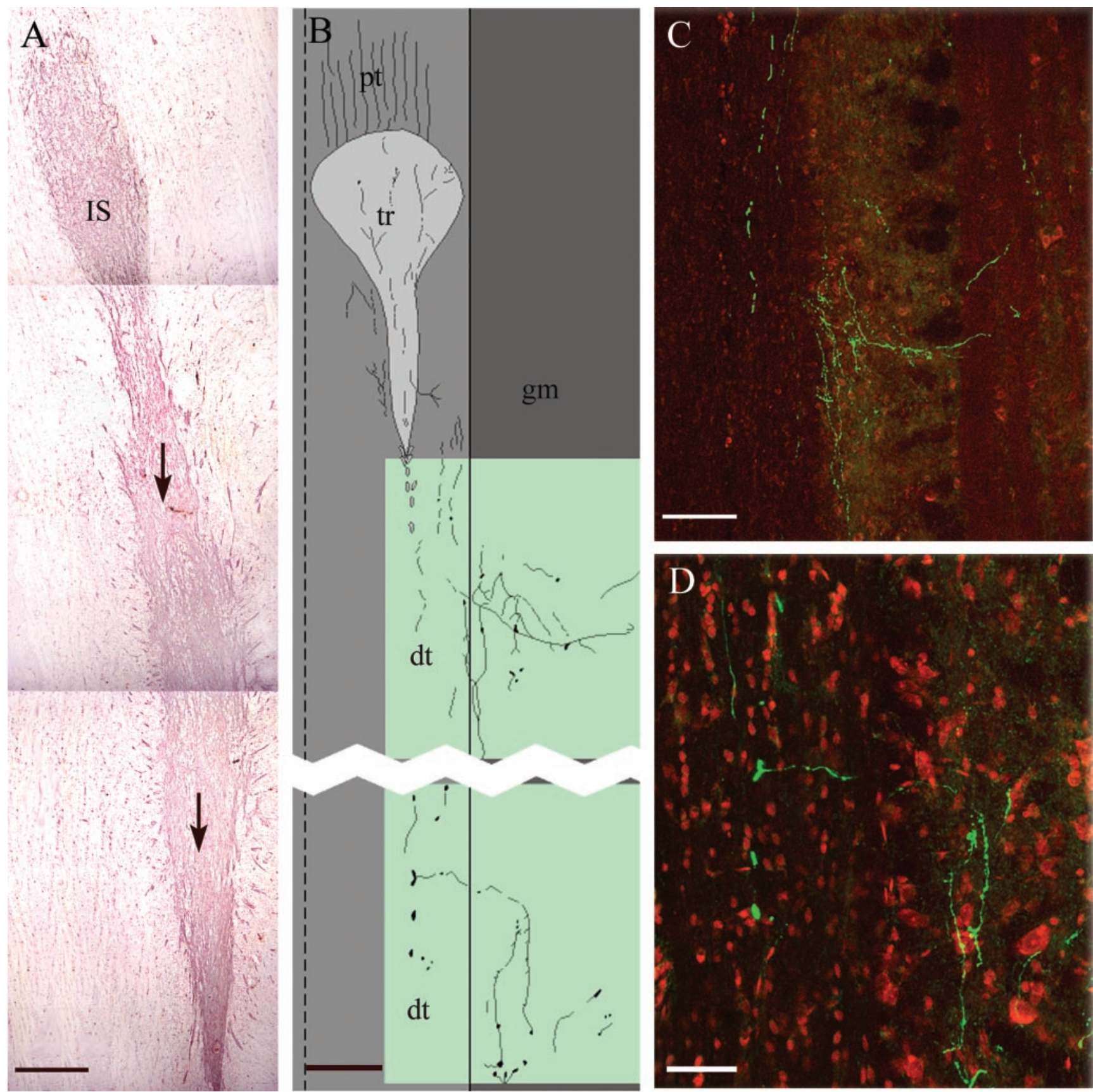

Figure 6. A, Montage of three horizontal 10- $\mu \mathrm{m}$-thick cryostat sections showing the selective migration (arrows) of the intensely FN-positive transplanted cells from the injection site (IS) caudally for $7 \mathrm{~mm}$ along the distal CST. The counterstain is neutral red. B, Camera lucida drawing of the position of BD-labeled CST axons passing from the proximal tract (pt) through a transplant (tr) and caudally into the distal CST (dt) and forming arborizations in the adjacent gray matter (gm) lateral to the tract. Dashed line, Midline; solid line, border between CST and adjacent gray matter. Green shading shows the areas photographed in Cand D. C, D, Confocal images showing BD anterogradely labeled CST axons (green) passing caudally through the distal CST, with collaterals traveling medially at right angles and generating a terminal arborization in the gray matter. Horizontal $60-\mu \mathrm{m}$-thick cryostat sections caudal to an OEC transplant placed in a complete CST lesion. The counterstain is propidium iodide (red). Scale bars: $A, 500 \mu \mathrm{m} ; B, C, 250 \mu \mathrm{m} ; D, 100 \mu \mathrm{m}$. Survival times ( $A-D)$ are 8 weeks after lesion plus 8 weeks after transplant.

sion/transplant site, the BD-labeled axons continued as straight, primarily unbranched fibers for up to $11 \mathrm{~mm}$ through the caudal part of the CST, emitting collateral branches that left at right angles and traveled for up to $400 \mu \mathrm{m}$ in a lateral direction into the adjacent gray matter, where they broke up into arborizations (Fig. $6 B-D$ ).

In 10 rats studied in cross-section histology, $\mathrm{P} 0$ immunohistochemistry for peripheral myelin clearly showed that the axons passing through the transplants were myelinated by OECs (Fig. 7). The P0 staining in cross-section enables us to count the total number of fibers crossing the transplants (Fig. $7 B, C$ ). For seven rats, the mean and SE was $526 \pm 58$.

\section{Discussion}

Delayed repair will be a necessity in any future clinical trials, if only because the unpredictability of "spontaneous" repair means the prognosis for any individual spinal-injured patient may not be clear for up to 1 year after injury. In the present study, we have attempted to provide a model of a delayed transplantation para- 


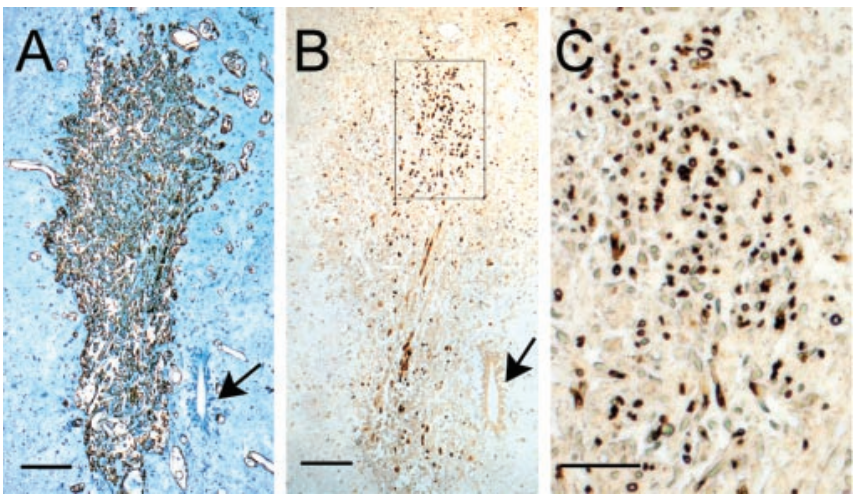

Figure 7. $A$, Cross-section showing a transplant stained with anti-FN antibody in the CST (compare Fig. 5A). B, Adjacent section stained with anti-P0 antibody to show the regenerating axons ( $~ 200-300$ in this case) myelinated by 0EC (peripheral-type) myelin. C, Enlargement of the area outlined in $B$. Survival times are 8 weeks after lesion plus 8 weeks after transplant. Arrows, Central canal. A two micrometer semithin section is counterstained with methylene blue and Azur II. Scale bars: $A, B, 100 \mu \mathrm{m} ; C, 50 \mu \mathrm{m}$.

digm. The most important conclusion of the present observations is that the previously reported reparative effect of immediate transplantation OECs on DFR (Li et al., 1997) can still be obtained when the transplantation is delayed for 8 weeks after the lesion.

\section{The effect of CST lesions on DFR}

The present results indicate that DFR is abolished for at least 6 months after unilateral destruction of the rat dorsal CST at the top cervical level. This loss of DFR is not associated with either paralysis or any obvious weakness of the affected forepaw. Moreover, motivation is clearly intact, because directed retrieval movements of the affected forepaw are readily initiated, but they are abortive. The failure seems to be in the ability of the proximal musculature to extend the forelimb through the slit and place it in a position in which the wrist flexors would be able to bring the paw into contact with the food pellet. There is no obvious asymmetry in the use of the forelimbs in walking, grooming, or in handling the food once it has been grasped. The selective sensitivity of the proximal muscles to CST lesions fits with an anatomical and electrophysiological study (Küchler et al., 2002) that showed that, in contrast to the CST, the rubrospinal tract has a monosynaptic projection to the somata and major dendrites of the cervical motoneurons innervating the distal forelimb muscles but has a less direct influence over the more proximal muscles.

A complex coordinated movement such as DFR (together with all the other necessary adjustments of body position, balance, and posture needed to make the arm and paw retrieval effective) involves activity of many supraspinal and intraspinal pathways. Although a number of different ascending and descending spinal tracts certainly contribute components to the overall performance of DFR (McKenna and Whishaw, 1999; Weidner et al., 2001), our present use of small circumscribed lesions confirms our previous finding that severance of the dorsal CST that does not affect other tracts, such as the rubrospinal tract (in the lateral columns) or the ventral CST (in the ventral columns), and that causes only minor and variable damage to the ascending (sensory) dorsal columns nevertheless completely abolishes ipsilateral DFR, and there is no return of this function for up to 6 months.

After partial spinal cord lesions, it is clear from both animal and human studies that major functional recovery can occur in the absence of regeneration of the severed connections. The mechanism of this recovery probably involves a combination of formation of new connections by sprouting and transfer of function between pathways (Raineteau et al., 2002). Nonetheless, with larger lesions, there always seems to be an element of function that is permanently lost. Thus, although a patient may have an injury in which some functions are spared, he may still be left with severe and intractable disabilities. Presumably, the tracts that have been severed mediate a unique type of information that sprouting or relearning by the remaining tracts is unable to restore. This model seems to fit the obligatory relationship between the dorsal CST as a whole and DFR.

However, our results with incomplete lesions of the CST indicate that within the CST specificity is less fixed. After partial lesions of the CST, DFR recovers at a rate, and to a degree, proportional to the number of spared CST fibers. This suggests that the CST does not have specific subgroups of fibers dedicated to specific functions. The delayed return of DFR after incomplete CST lesions shows that, when partially destroyed, the surviving part of the CST is capable of reestablishing functions with even very small numbers of surviving fibers (Fig. $2 H$ ).

\section{Restoration of function by delayed transplants}

Recovery of ipsilateral DFR after complete unilateral CST lesions occurs only when OECs are transplanted into the lesion site. Transplants misplaced in the dorsal columns, although only 1-2 $\mathrm{mm}$ away from the lesion site, did not cause recovery of function (Fig. 5B). Although we have seen transplanted OECs migrating for up to $11 \mathrm{~mm}$ within the distal CST, their migratory ability is not sufficient to cross tissue boundaries such as those between adjacent white matter tracts. To be effective, the cells must be placed in direct contact with the tract to be repaired.

Additional anatomical studies will be needed to elucidate the mechanism by which the transplanted OECs mediate return of function. For us, one of the most attractive ideas is that the functions are restored by regeneration of severed CST fibers. Certainly, in this and the previous study (Li et al., 1997), we have seen instances of $\mathrm{BD}$ anterogradely labeled CST fibers crossing the transplant and traveling through the distal CST to their normal destinations (Fig. $6 B-D$ ). P0 staining shows that these fibers are few in number, $526 \pm 58$ myelinated fibers (Fig. $7 \mathrm{~B}, C$ ), which is only $\sim 1 \%$ of the total number of $\sim 50,000$ CST myelinated fibers (Li et al., 1997). That such small numbers of fibers can mediate useful return of function is also indicated by the observation that in untransplanted rats in which the lesions spare only $\sim 1 \%$ of the CaM-II-positive CST fibers (Fig. $2 \mathrm{H}$ ), DFR recovers spontaneously. It must also be noted that although the terminal distribution of the regenerating fibers is into the same area as normal, we have no indication of the extent to which the cortical somatotopic map is restored, nor whether fibers can regenerate much further, for example to lumbar levels.

It remains a possibility that transplanted OECs may induce recovery of function, at least in part, by stimulation of uncut fibers or by humoral mechanisms, possibly associated with the intense vascularization of the transplants or remyelination of undetected spared demyelinated fibers. But, whatever the mechanism, the functional outcome is clear: only transplants bridging the lesion are able to restore function. In terms of future human therapy, the encouraging aspects of the present findings are: (1) that recovery of function is induced by transplants in situations in which no spontaneous recovery otherwise occurs; and (2) that the beneficial reparative effects of donor OEC transplants can still 
be obtained if transplantation is delayed for 2 months after the CST lesions in rats.

\section{References}

Brandt HM, Apkarian AV (1992) Biotin-dextran: a sensitive anterograde tracer for neuroanatomic studies in rat and monkey. J Neurosci Methods 45:35-40.

Coumans JV, Lin TTS, Dai HN, MacArthur L, McAtee M, Nash C, Bregman BS (2001) Axonal regeneration and functional recovery after complete spinal cord transection in rats by delayed treatment with transplants and neurotrophins. J Neurosci 21:9334-9344.

Imaizumi T, Langford KL, Waxman SG, Greer CA, Kocsis JD (1998) Transplanted olfactory ensheathing cells remyelinate and enhance axonal conduction in the demyelinated dorsal columns of the rat spinal cord. J Neurosci 18:6176-6185.

Küchler M, Fouad K, Weinmann O, Schwab ME, Raineteau O (2002) Red nucleus projections to distinct motor neuron pools in the rat spinal cord. J Comp Neurol 448:349-359.

Li Y, Field PM, Raisman G (1997) Repair of adult rat corticospinal tract by transplants of olfactory ensheathing cells. Science 277:2000-2002.

Li Y, Field PM, Raisman G (1998) Regeneration of adult rat corticospinal axons induced by transplanted olfactory ensheathing cells. J Neurosci 18:10514-10524.

Lu J, Féron F, Mackay-Sim A, Waite PME (2002) Olfactory ensheathing cells promote locomotor recovery after delayed transplantation into transected spinal cord. Brain 125:14-21.
McKenna JE, Whishaw IQ (1999) Complete compensation in skilled reaching success with associated impairments in limb synergies, after dorsal column lesion in the rat. J Neurosci 19:1885-1894.

Plant GW, Christensen CL, Oudega M, Bunge MB (2003) Delayed transplantation of olfactory ensheathing glia promotes sparing/regeneration of supraspinal axons in the contused adult rat spinal cord. J Neurotrauma 20:1-16.

Raineteau O, Fouad K, Bareyre FM, Schwab ME (2002) Reorganization of descending motor tracts in the rat spinal cord. Eur J Neurosci 16:1761-1771.

Ramón-Cueto A, Plant GW, Avila J, Bunge MB (1998) Long-distance axonal regeneration in the transected adult rat spinal cord is promoted by olfactory ensheathing glia transplants. J Neurosci 18:3803-3815.

Ramón-Cueto A, Cordero MI, Santos-Benito FF, Avila J (2000) Functional recovery of paraplegic rats and motor axon regeneration in their spinal cords by olfactory ensheathing glia. Neuron 25:425-435.

Terashima T, Ochiishi T, Yamauchi T (1994) Immunohistochemical detection of calcium/calmodulin-dependent protein kinase II in the spinal cord of the rat and monkey with special reference to the corticospinal tract. J Comp Neurol 340:469-479.

Weidner N, Ner A, Salimi N, Tuszynski MH (2001) Spontaneous corticospinal axonal plasticity and functional recovery after adult central nervous system injury. Proc Natl Acad Sci USA 98:3513-3518.

Zurita M, Vaquero J, Oya S, Montilla J (2001) Functional recovery in chronic paraplegic rats after co-grafts of fetal brain and adult peripheral nerve tissue. Surg Neurol 55:249-254. 